\title{
R2 - Avaliação da utilização de ensaio imunocromatográfico para o diagnóstico e estudo de prevalência da hepatite $\mathbf{A}$
}

Camilla Rodrigues de Almeida Ribeiro ${ }^{1 *}$; Luciane Almeida Amado ${ }^{1}$; Renata Tourinho Santos ${ }^{1}$; Lyana Rodrigues Lima ${ }^{1}$; Juliana Melgaço ${ }^{1}$; Adilson José de Almeida $^{2}$; Lia Laura Lewis Ximenez ${ }^{3}$; Vanessa Salete de Paula ${ }^{1}$.

1 - Laboratório de Desenvolvimento Tecnológico em Virologia, Instituto Oswaldo Cruz, Fundação Oswaldo Cruz;

2 - Ambulatório de Hepatites Virais, Instituto Oswaldo Cruz, Fundação Oswaldo Cruz, Rio de Janeiro, Brasil e Hospital Universitário Gaffrée e Guinle, Setor de Hematologia, Universidade Federal do Estado do Rio de Janeiro;

3 - Ambulatório de Hepatites Virais, Instituto Oswaldo Cruz, Fundação Oswaldo Cruz;

\section{Introdução:}

A hepatite A é uma doença infecciosa aguda, e embora seja auto-limitada é uma significante causa de morbidade e perdas socioeconômicas em muitas partes do mundo. Em países em desenvolvimento, o acentuado declínio na taxa de incidência, resultante das melhorias nas condições sanitárias, contribuiu para a redução da exposição ao vírus da hepatite A (HAV) durante a infância e o aumento da incidência em idades mais avançadas aumentando o risco de morbidade e mortalidade pela doença. Com base nesses dados epidemiológicos e na importância mundial da hepatite $\mathrm{A}$, ensaios rápidos e práticos devem ser avaliados (sensibilidade, especificidade, reprodutibilidade e valores preditivo), para facilitar o diagnóstico dessa patologia, possibilitando o tratamento precoce e evitando a sua transmissão.

\section{Objetivo:}

Avaliar a eficiência e eficácia de um ensaio imunocromatográfico comercial (teste rápido) para a diagnóstico da hepatite A e estudo de prevalência em diferentes grupos de estudo.

\section{Metodologia:}

Foram avaliados parâmetros como a sensibilidade, especificidade, valores preditivo positivo e negativo, reprodutibilidade e repetitividade do teste rápido para detecção de anti-HAV IgM e anti-HAV IgG em amostras de soro previa- 
mente identificadas como reagentes e não reagentes. Amostras de pacientes ( $\mathrm{n}$ total $=342$ ) de diferentes grupos foram coletadas e submetidas ao teste rápido SD BIOLINE HAV IgG/IgM (Standard Diagnostics, Corea), todos os resultados do teste rápido foram comparados com os resultados do teste imunoenzimático (Elisa) ETI-AB-HAVK PLUS (Diasorin, Itália) e Biokit (Biokit, S.A. Barcelona, Espanha), que é padrão ouro para o diagnóstico sorológico da hepatite A.

\section{Resultados:}

O limite de detecção do teste rápido para anticorpos Anti-HAV IgG foi de 10-2 e do teste padrão-ouro (Elisa) foi de 10-10 e para para anticorpos Anti-HAV IgM o teste rápido demostrou limites de detecção iguais ou superiores ao padrão-ouro. O teste rápido não demostrou reatividade cruzada com anticorpos provenientes de outros agentes infecciosos e demostrou ter uma taxa de detecção menor comparada ao Elisa em todos os grupos, para ambos os marcadores. Para a detecção do anti-HAV IgG o teste não apresentou boa repetitibilidade e reprodutibilidade. Os níveis de concordância utilizando o índice Kappa (k) foram considerados de fracos a bons nos grupos testados para o marcador IgG e de excelentes a bons para os grupos testados com marcador IgM e a sensibilidade e a especificidade do teste rápido para detecção de anti-HAV IgM foi de 94,74 (IC 95\%; 88,14-98,27) e 99,05 (IC 95\%; 94,81- 99,98) e para anti-HAV IgG foi de 69,61 (IC 95\%; 62,80- 75,84) e 66,30 (IC 95\%; 55,70-75,83), respectivamente.

\section{Conclusão:}

Comprovamos que o teste mostrou ser adequado para o diagnóstico da hepatite A em casos de surtos e casos esporádicos para detecção de anticorpos IgM. No entanto, para detecção de infecção passada e resposta vacinal, o teste precisa ser aprimorado.

\section{Palavras-Chave: Hepatite A, teste rápido}

